\title{
Neoadjuvant and Adjuvant Chemotherapeutic Strategy of Colorectal Mixed Adeno- Neuroendocrine Carcinomas
}

\author{
Anita Michael ${ }^{1}$, Debashis K. Nath ${ }^{2}$ \\ 1. Internal Medicine, PSG Institute of Medical Sciences and Research, Coimbatore, IND 2. Internal Medicine, Queen \\ Elizabeth Hospital Kings Lynn, King's Lynn, GBR
}

Corresponding author: Anita Michael, anitamichael13@gmail.com

\begin{abstract}
Mixed adeno-neuroendocrine carcinomas (MANEC) is a rare pathological diagnosis characterized by the presence of both adeno-carcinomatous and neuroendocrine differentiation with each component comprising $30 \%$ of the tumor. This literature review is aimed at the extraction of all existing clinical studies and reviews on colorectal MANEC so as to ensure that a suitable chemotherapeutic regimen is chosen to improve survival outcomes and prognosis of the disease. Parallel search strategies were employed to extract past 10 years articles from PubMed, PubMed Central and Google Scholar databases. A total of 30 records consisting of one clinical trial, five retrospective cohort studies, one case control study, one case series, 16 case reports and six review papers were shortlisted. Chemotherapeutic regimens that were administered as an adjuvant and a neoadjuvant therapy were analyzed with their survival outcomes. The overall survival rate of those administered with neoadjuvant and adjuvant therapy can be as high as $57.4 \%$ and $69 \%$, respectively. Multiple chemotherapeutic regimens were employed in colorectal MANEC and superiority of one regimen over the other can't be established. Any drug or combination of drugs that is responsive against either of the MANEC components is found to be effective against the tumor. However, excellent responsiveness has been found with 5 -fluorouracil regimens as a neoadjuvant therapy and platinum-based combinations as an adjuvant therapy. XELOX, streptozocin and S1 regimens also prove to be drugs of choice in aggressive and metastasized disease conditions. Our analysis allows for improved chemotherapeutic management of individuals with colorectal MANEC and establishes an increased potential for use of streptozocin therapy in the clinical setting. However, newer drugs like amrubicin require further research prior to describing its efficacy in colorectal MANEC.
\end{abstract}

Review began 06/19/2021 Review ended 07/12/2021 Published 07/26/2021

(๑) Copyright 2021

Michael et al. This is an open access article distributed under the terms of the Creative Commons Attribution License CC-BY 4.0., which permits unrestricted use, distribution, and reproduction in any medium, provided the original author and source are credited.
Categories: Internal Medicine, Oncology

Keywords: manec, colorectal, neoadjuvant, adjuvant, treatment, management

\section{Introduction And Background}

Mixed adeno-neuroendocrine carcinomas (MANEC) as defined by the World Health Organization (WHO) in 2010, is distinguished by the simultaneous presence of both adeno-carcinomatous (epithelial) and neuroendocrine differentiation, such that each component represents at least 30\% of the tumor [1]. Table 1 establishes the classification of MANEC with its histologic appearance as illustrated by La Rosa et al. [2]. 


\section{Cureus}

Classification of mixed

adenocarcinoma-

neuroendocrine

neoplasms

High grade malignant

MANEC

Intermediate grade

malignant MANEC

Low-grade malignant

MANET
Composition (histology)

Prognosis

Mixed adenomatous / carcinomatous tumor with poorly differentiated neuroendocrine carcinomas.

Mixed adenocarcinoma neuroendocrine tumor that comprises of an exocrine component which is an adenocarcinoma and a neuroendocrine component which is NET-G1 or NET-G2.

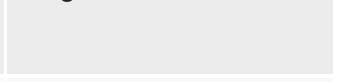

Worst Prognosis

Overall Survival (OS) is greater than High-Grade Malignant tumors.

Well differentiated neuroendocrine and exocrine components.

Good Prognosis

\section{TABLE 1: Classification, histological appearance and prognosis of MANEC.}

MANEC: Mixed adeno-neuroendocrine carcinomas, MANET: Mixed adeno-neuroendocrine tumors, NET-G1: Grade 1 neuroendocrine tumor, NETG2: Grade 2 neuroendocrine tumor.

Positive immuno-histochemical staining for chromogranin (CgA), synaptophysin (Syn) and CD56 is used to validate the presence of a neuroendocrine component in MANEC. Colorectal neuroendocrine carcinoma (NEC) by itself is a rare pathological diagnosis and constitutes less than $2 \%$ of all malignancies of the colon and rectum [3]. The management of colorectal MANEC cannot be attributed to the therapeutic strategy of adenocarcinomas, due to the mixed histological presentation. It is, however, influenced by the type and degree of neuroendocrine differentiation and has a better prognostic score compared to those with neuroendocrine carcinomas [4].

'National comprehensive cancer network guidelines' does not provide a specific management strategy for MANEC, however, a complete surgical resection of both the colorectal tumor and metastases followed by chemotherapeutic management (adjuvant) is the widely accepted norm [5]. Neoadjuvant chemotherapy is reported to reduce the risk of metastasis and prolong disease-free survival rates in MANEC [6]. Qiu et al. report that surgical resection with a deliberate adjuvant chemotherapeutic management has a greater survival and a better prognosis [7].

With limited literatures and statistical evidences on colorectal MANEC, it is not possible to estimate the level of similarities to its adenocarcinoma and neuroendocrine counterparts. Moreover, with recognition as a distinct clinical entity, it is imperative that we compile all neoadjuvant and adjuvant chemotherapeutic modalities used and recorded so as to ensure a better management and survival with this rare pathological entity.

\section{Review}

\section{Method and result}

Objective and Study Design

Our study design revolves around the extraction of existing neoadjuvant and adjuvant chemotherapeutic strategies to assist in resection and in improving survival. On a secondary perspective, this literature review also hopes to accumulate all observational and case reports to provide with a knowledge of prior experience in chemotherapeutic management of colorectal MANEC.

Retrospective and prospective cohorts, case-control studies, observational studies and case reports on patients diagnosed with colorectal MANEC as well as review papers describing a general and specific management of colorectal MANEC are considered. A search strategy of 'MANEC' automatically excludes all articles prior to 10 years, given that the term MANEC was defined as a distinct entity in 2010. However, an additional search filter was placed at past 10-year literatures (2010-2020) to avoid irrelevant search results. Full-text papers were evaluated with language restrictions bounded to English to ensure ease of analysis and tabulation while studies on animal models and meta-analysis were excluded from the study. Pediatric age groups were also excluded from the study due to inconsistent approach of management outline between pediatric and adult (19+) cohorts.

This review has been carried out with a scrupulous attention to the clinical and therapeutic approach and publications that do not justify this approach are excluded. An extensive methodology allowed for a strategic elimination of overlapping literatures and a thorough evaluation of existing literatures that meet with the above criterion. 


\section{Cureus}

Search Strategy and Data Collection

Literatures published on and before January 2021 were assessed following the application of a limited number of keywords 'MANEC', 'treatment' and 'colorectal' to ensure that the loss of quality publications is at a minimum. With the availability of limited articles on MANEC, it is imperative that the data procured is maximized. Parallel strategies were used in the evaluation of articles in 'PubMed', 'PubMed Central' and 'Google Scholar' databases. Data collection using the application of MESH keywords was not performed due to a significant decrease in the number of quality articles based on which analysis would then be irrelevant.

Management outlines were not amplified among individuals of varied age (provided they were above 19) and gender. At the same time, tumor advancement, survival, and the type of neoadjuvant and adjuvant chemotherapy is evaluated simultaneously so as to ensure unbiased data collection.

Tumor resection is taken to be a necessity and only chemotherapy administered as a subsidiary treatment before or after surgery is read and described. Hence, margin for error is minimized while maximizing the approach towards this unusual diagnosis.

Search Result and Analysis

An extensive screening and analysis were performed to identify relevant articles in PubMed, PubMed Central and Google Scholar databases. Overlapping or duplicate articles in the three databases were excluded and only those literatures that concede with the objective of the review paper were analyzed and reported. Table 2 provides an insight into the parallel screening process across the three databases on application of inclusion and exclusion criteria.

\begin{tabular}{|c|c|c|}
\hline Keywords applied & Total records & Records selected \\
\hline \multicolumn{3}{|l|}{ PubMed database } \\
\hline MANEC & 211 & 174 \\
\hline MANEC, treatment & 121 & 95 \\
\hline MANEC, treatment, colorectal & 17 & 15 \\
\hline \multicolumn{3}{|l|}{ PubMed Central (PMC) database } \\
\hline MANEC & 365 & 321 \\
\hline MANEC, treatment & 342 & 309 \\
\hline MANEC, treatment, colorectal & 155 & 154 \\
\hline \multicolumn{3}{|l|}{ Google Scholar database } \\
\hline MANEC & 7590 & 1470 \\
\hline MANEC, treatment & 2150 & 1230 \\
\hline MANEC, treatment, colorectal & 685 & 524 \\
\hline
\end{tabular}

\section{TABLE 2: Primary screening process of article selection by applying inclusion and exclusion criteria}

MANEC: Mixed adeno-neuroendocrine carcinomas

Primary screening process of article selection yields a total of 693 literatures across three databases. These articles are chosen on the application of basic inclusion and exclusion criteria. A secondary screening process is performed to remove overlapping or duplicate articles, research based on animal models, secondary data analysis (meta-analysis), literatures where data extraction is not possible and those which are irrelevant to the objective of this review. Further, papers which outline a general management of MANEC are also manually chosen and analyzed with a motive to provide potential chemotherapeutic modalities in the neoadjuvant and adjuvant management of colorectal MANEC. Figure 1 summarizes the secondary process of screening and literature selection. 


\section{Cureus}

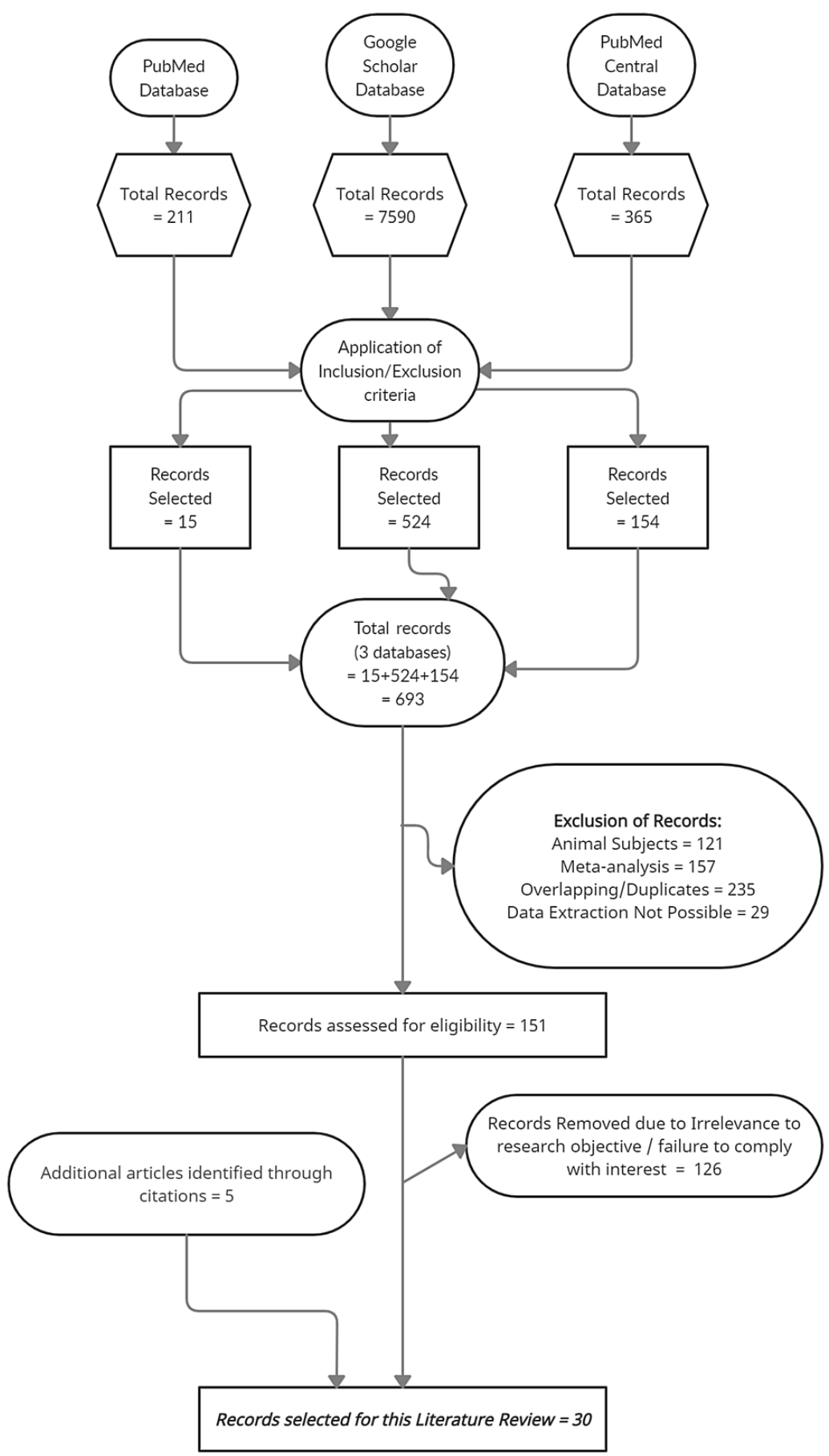

FIGURE 1: Preferred reporting items for systematic reviews and metaanalyses (PRISMA) flow diagram to summarize the process of screening and literature selection

A total of 30 articles are analyzed which include 16 case reports [8-23], one clinical trial [24], five retrospective cohort studies [6, 25-28], one case control study [29], one case series [30] and six reviews $[2,3,5,7,31,32]$. Table 3 provides a summary of the 16 case reports analyzed in an attempt to throw light onto the individual clinical presentation of patients with MANEC and the chemotherapeutic regimens administered. Tumor staging was based on American Joint Committee on Cancer (AJCC) classification. 


\section{Cureus}

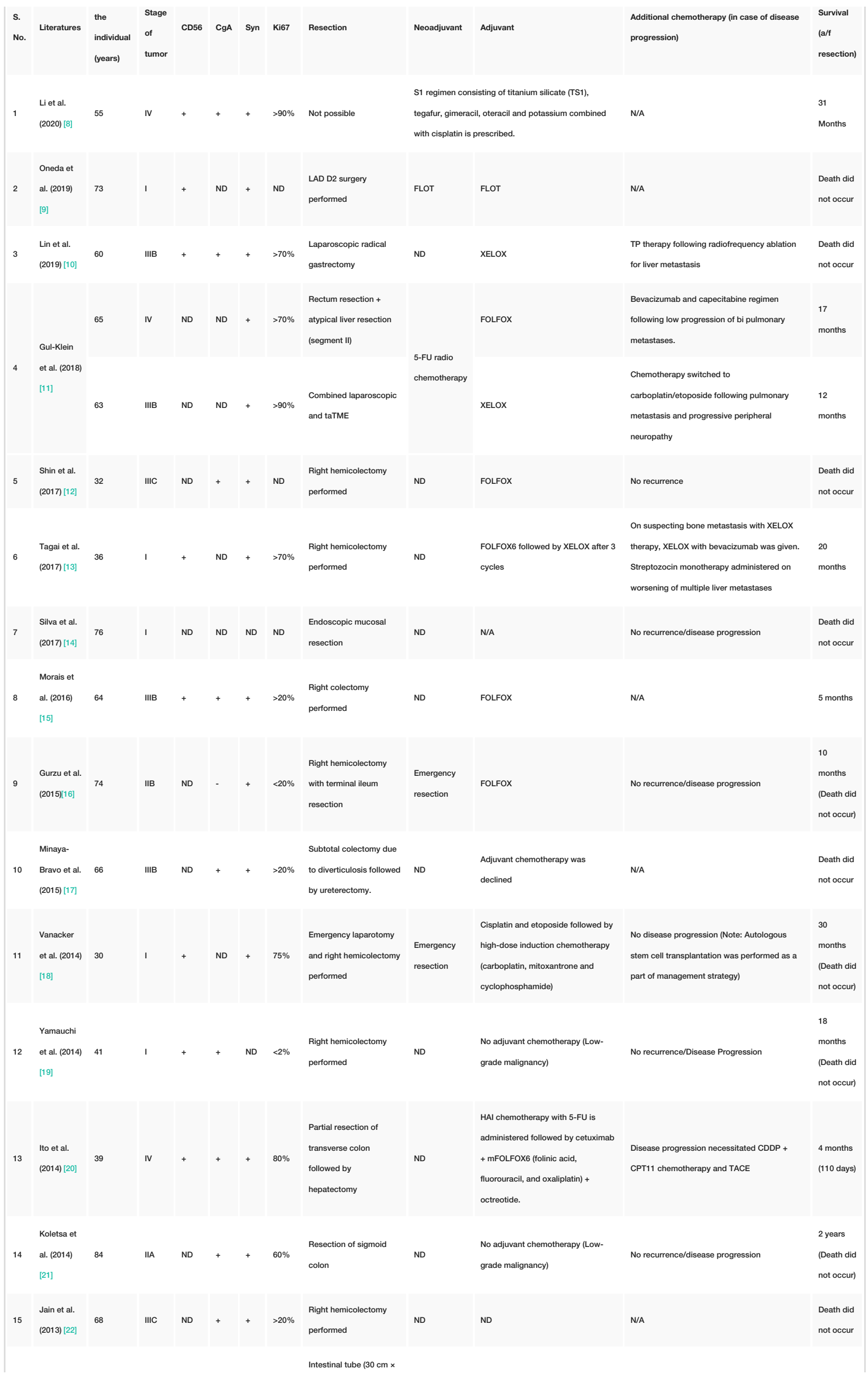




\section{Cureus}
16 Liu et
68
IIA
$13 \mathrm{~cm} \times 4.5 \mathrm{~cm})$ and
appendix on ileocecal
ND
No recurrence/disease progression

\section{TABLE 3: Summary of case reports analyzed in this literature review}
NC: Neuroendocrine component, CgA: Chromogranin A, Syn: Synaptophysin, a/f: After, N/A: Not applicable, +/-: Positive/Negative, ND: Not described, LAD: Gastric lymphadenectomy, FLOT: Fluorouracil, leucovorin, oxaliplatin and docetaxel, XELOX: Capecitabine and oxaliplatin, TP: Paclitaxel and cisplatin, 5-FU: 5-fluorouracil, FOLFOX: Leucovorin, fluorouracil and oxaliplatin, taTME: Transanal total mesorectal excision, HAI: Hepatic artery infusion, CDDP: Cisplatin, CTP-11: Irinotecan, TACE: Trans arterial chemoembolization.

S1 regimen consisting of titanium silicate, tegafur, gimeracil, oteracil and potassium is used in the management of MANEC in conditions where resection is not possible or indicated. S1 regimen combined with cisplatin is considered to be effective with survival up to 31 months [8].

In cases where resection is indicated, neoadjuvant chemotherapy is given less importance by practitioners as opposed to adjuvant therapy which is seen almost as a necessity to prevent relapse or recurrence. It is not feasible in stage one tumors with low proliferative rates [12] and cases where diagnosis of MANEC is coupled with emergency resection $[9,11]$. In the advent of administration, an antimetabolite, commonly fluorouracil and its derivatives is prescribed with an objective to inhibit tumor progression or growth prior to resection $[9,11]$.

Stage I MANEC can exhibit high proliferative rates (Ki67 index >70\%), progressive metastases and a possible death, hence frequent examination and changes in chemotherapeutic drugs for all stages of tumor are essential to increase tumor response [13]. Pulmonary and hepatic metastases necessitate a switch in chemotherapeutic regimens $[10,11]$. Streptozocin monotherapy was found to be effective in the treatment of unresponsive hepatic metastases [13]. Autologous stem cell transplantation was also used as a management strategy along with adjuvant chemotherapy in the prevention of disease progression [18].

Patients with stage IV tumors show a survival of up to 17 months [11] following resection and 31 months on administration of S1 regimen [8]. Individuals with stage I tumors can live up to 30 months or more [18], however, the survival depends equally on the rate of proliferation of tumor (Ki67 index) as that of the tumor stage. Table 4 describes the summary of clinical studies analyzed with an insight into the overall survival rate of individuals with colorectal MANEC. 


\section{Cureus}

\begin{tabular}{|c|c|c|c|c|c|c|c|}
\hline $\begin{array}{l}\text { S. } \\
\text { No. }\end{array}$ & Literatures & $\begin{array}{l}\text { Study } \\
\text { Design }\end{array}$ & $\begin{array}{l}\text { Sample } \\
\text { Size }\end{array}$ & $\begin{array}{l}\text { Follow-Up } \\
\text { (Months) }\end{array}$ & Chemotherapy analyzed & $\begin{array}{l}\text { OS } \\
(\%)\end{array}$ & $\begin{array}{l}\text { ORR } \\
(\%)\end{array}$ \\
\hline 1 & $\begin{array}{l}\text { Araki et al. (2016) } \\
\text { [24] }\end{array}$ & Cl & 19 & 11.5 & Amrubicin administration & - & 18.8 \\
\hline 2 & Ma et al. (2020) [6] & $\mathrm{RC}$ & 69 & 61 & NAC (EP/IP vs. No NAC) & 57.4 & 65 \\
\hline 3 & Lin et al. (2020) [25] & $\mathrm{RC}$ & 804 & 62 & AC (comparison between 5-FU, no 5-FU and no AC) & 53.2 & - \\
\hline 4 & $\begin{array}{l}\text { Song and Yuan } \\
\text { (2019) [26] }\end{array}$ & $\mathrm{RC}$ & 131 & 36 & Chemotherapy compared to no chemotherapy & 37.68 & - \\
\hline 5 & $\begin{array}{l}\text { van der Veen et al. } \\
\text { (2018) [27] }\end{array}$ & $\mathrm{RC}$ & 49 & 60 & $\begin{array}{l}\text { NAC (between platinum, non-platinum and no NAC) AC } \\
\text { (ECC, EP, XELOX and no AC) }\end{array}$ & 39 & - \\
\hline 6 & $\begin{array}{l}\text { Komatsubara et al. } \\
\text { (2016) [28] }\end{array}$ & $\mathrm{RC}$ & 12 & 36 & $\mathrm{AC}$ (5-FU and other $\mathrm{AC}$ regimens) & 27 & - \\
\hline 7 & $\begin{array}{l}\text { Watanabe et al. } \\
\text { (2016) [29] }\end{array}$ & CC & 42 & 40 & $\begin{array}{l}\text { AC (FOLFOX, FOLFOX + cetuximab, XELOX, XELOX + } \\
\text { bevacizumab) }\end{array}$ & 69 & 40 \\
\hline 8 & $\begin{array}{l}\text { Dulskas } \\
\text { and Pilvelis (2019) } \\
{[30]}\end{array}$ & $\mathrm{CS}$ & 9 & 17 & Chemotherapy, radiotherapy or both administered & 55.6 & - \\
\hline
\end{tabular}

\section{TABLE 4: Summary of clinical studies analyzed in this literature review}

OS: Overall survival, ORR: Overall response rate, CT: Clinical trial, RC: Retrospective cohort study, CC: Case control study, CS: Case Series, NAC: Neoadjuvant chemotherapy, EP: Etoposide and cisplatin, IP: Cisplatin and paclitaxel, AC: Adjuvant chemotherapy, 5-FU: 5-fluorouracil, ECC: Epirubicin, cisplatin and capecitabine, XELOX: Capecitabine and oxaliplatin, FOLFOX: Leucovorin, fluorouracil and oxaliplatin.

The overall survival rate of individuals treated with adjuvant or neoadjuvant chemotherapy is found to be significantly higher than those treated with only resection. An overall survival rate of up to $57.4 \%$ and $69 \%$ in those administered with neoadjuvant and adjuvant therapy respectively can be seen in individuals with colorectal MANEC. Platinum-based regimen etoposide + cisplatin and cisplatin + paclitaxel (EP/IP) seems promising as a first line drug with a response rate of $65 \%$. A new drug amrubicin is under clinical trials and reports a response rate of $18.8 \%$.

The maximum number of individuals employed in the study stands at 804 with the minimum number at nine. 5-fluorouracil-based compounds, XELOX, FOLFOX and platinum-based combinations particularly etoposide and cisplatin have been studied extensively among the cohort.

\section{Discussion}

Multiple treatment options are considered and a variety of regimens have been prescribed in the treatment of colorectal MANEC. No regimen is considered to be superior over the other. Nevertheless, it is important to note that the duration of survival and response to different regimens greatly varies within the population.

\section{S1 Regimen}

Doublet chemotherapy consisting of tegafur, gimeracil, oteracil and potassium is a common adjuvant therapy in countries such as Japan. It can also be administered in combination with cisplatin as an effective neoadjuvant therapy [33]. S1 regimen is commonly administered in advanced or stage IV colon cancers and MANEC where resection is not indicated $[8,33]$. However, it is also seen as a gold standard adjuvant therapy in stage II or III gastric cancers and in hepatic metastases [34]. Anemia, leukopenia and neutropenia are commonly observed adverse drug reactions. However, it is seen as a relatively safer option with an optimal dose reduction following curative resection in stage III gastric cancers. $74.2 \%$ of individuals who participated in the study have completed the regimen [35].

A combination regimen of S1 along with cisplatin, paclitaxel, docetaxel and irinotecan is recommended to further improve survival outcomes [32]. While there has been limited study on the use of this regimen in MANEC, a positive response in gastric carcinoma shows potential for its use by targeting the adenocarcinomatous component. 
The XELOX regimen uses capecitabine and oxaliplatin as an adjuvant therapy in the treatment of individuals with gastric carcinomas and preferably in those with hepatic metastases. National comprehensive cancer network (NCCN) guidelines recommends its use in individuals following R0-D2 resection in gastric carcinomas [36]. A median overall survival (OS) rate of 19.8 months was observed with decreased incidences of Grade III or IV toxicities. Adverse effects were mild and include nausea, vomiting and diarrhea with occasional cases of paresthesia and peripheral neuropathy [37]. However, the response to XELOX therapy varies among individuals and it is recommended that preoperative treatment regimens and nutritional status of the individual is taken into consideration prior to administration [10].

\section{Fluorouracil-Based Treatment Regimens}

The recommended fluorouracil-based treatment regimens for MANEC include the 'FLOT' (fluorouracil, oxaliplatin and docetaxel) and 'FOLFLOX' (fluorouracil, leucovorin and oxaliplatin) regimens. Fluorouracilbased adjuvant therapy is targeted towards the adenoneuroendocrine component of the tumor [15]. Fluorouracil has an antimetabolic activity, thereby killing and inhibiting the growth of tumor cells. Hence, fluorouracil as a part of the FLOT regimen is also used commonly as a neoadjuvant therapeutic drug.

FLOT neoadjuvant therapy reports an increased overall survival (OS) of up to 50 months and an increased likelihood for the patient to undergo all stages of chemotherapy. Overall response rate (ORR) is considerable and is reported to be up to 55\%. While the incidences of leucopenia and neutropenia were less, a few individuals also reported grade III to IV hematological abnormalities and one patient dropped out of neoadjuvant therapy due to acute cerebral infarction [33]. With the use of FOLFOX therapy, duration of FLOT therapy can be reduced with comparable efficacies in stage III colon cancers. Six-year overall survival (OS) stands at $72.9 \%$ with the administration of FOLFOX regimen [38].

This review suggests FOLFOX over FLOT in patients with poor performance status due to the presence of significant adverse drug reactions but similar treatment response.

Platinum-Based Combination Regimens

Platinum-based combination regimens which are used as an adjuvant therapy for MANEC use cisplatin or carboplatin in combinations with etoposide, irinotecan and docetaxel. These have been described as TP (cisplatin + docetaxel), CDDP + CPT 11 (cisplatin + irinotecan), EP (cisplatin + etoposide) and carbo/etop (carboplatin + etoposide) regimens. These drugs have been administered in cases with high proliferative rates with increased risks of disease progression $[10,11,18,20]$. TP therapy can be used as a second line drug in liver metastases and EP therapy has been used in pulmonary metastasis of MANEC $[10,11]$. Platinum-based combination regimens, however, account for a number of toxicities with reports of renal dysfunction and febrile neutropenia [39] which makes it difficult for the administration of a second or third line chemotherapeutic drug into the clinical setting.

The involvement of neuroendocrine component in MANEC allows for a similar clinical behavior between MANEC and neuroendocrine tumors. Poorly differentiated gastrointestinal neuroendocrine tumors are highly aggressive and it is recommended that these tumors are treated with an EP regimen according to European neuroendocrine tumor society (ENETS) guidelines [36] and a carbo/etop or CDDP + CPT11 regimen according to national comprehensive cancer network (NCCN) guidelines [40]. Nordic guidelines 2014 for diagnosis and treatment of gastroenteropancreatic neuroendocrine neoplasms also recommend an EP or carbo/etop regimen in the treatment of neuroendocrine neoplasms [41].

EP regimen is considered to have a high response rate among individuals with colorectal high-grade metastatic neuroendocrine tumors, however, this is often followed with poor overall outcomes [31]. Hence, Vanacker et al. use autologous stem cell transplantation and high dose induction chemotherapy consisting of carboplatin, mitoxantrone and cyclophosphamide to improve survival outcomes of the individual. It reports that EP regimen alone following resection exhibits a maximum survival rate of not more than 17 months [18].

Platinum-based regimens are recommended if the Ki67 index of the neuroendocrine component is equal to or more than 55\%, which otherwise is considered to be ineffective [39]. No specific role is described for systemic adjuvant therapy in grade I and grade II neuroendocrine tumors, however, from a clinical perspective, we recommend its use in grade I and grade II highly proliferative tumors.

Streptozocin Monotherapy

'The guidelines committee of the Japan neuroendocrine tumor society' suggests the use of streptozocin, a cytotoxic agent with 5-fluorouracil or doxorubicin for the treatment of grade I and grade II neuroendocrine tumors [42]. It is also considered to be more effective as compared to monotherapy [43]. Tagai et al. report a partial response to streptozocin monotherapy on treatment of colorectal MANEC [13] and is also reported to show a response rate of about $25 \%$ in gastrointestinal neuroendocrine tumor patients [44]. 
Krug et al. report that $3 \%$ of individuals presented with renal failure and only $10 \%$ reported with grade III or IV toxicities [43]. The common adverse reactions reported with streptozocin therapy were mild and include vomiting, nausea and lethargy [44]. This limited adverse reaction also paves way for the safe administration of a third line chemotherapeutic drug in the setting. While no other reports of streptozocin therapy for the treatment of MANEC can be found, it is recommended for daily practice in pancreaticoduodenal and gastrointestinal neuroendocrine tumors [44], and thus can be safely assumed that it has the potential to be an effective treatment regimen in the management of colorectal MANEC.

Prognosis

A limited number of literatures makes it difficult to determine its prognosis. A median survival rate is estimated to lie between seven to 10 months following diagnosis and prognosis appears to be bleak [11]. However, benign and low grade malignant cases of MANEC show cure without disease progression and recurrence. Submucosal layer involvement of low-grade malignancies, proliferative rate of the tumor and the percentage of neuroendocrine component in the tumor determine its prognosis to some extent although a definite conclusion cannot be made [21].

Limitations

Only full text articles were included for an ease of accessibility and non-English literature was removed to avoid misinterpretation and translational error. This could have underestimated the available clinical data as a result of decreased sample size analysis. Multiple studies on MANEC were performed on animals, however, a significant difference in biological structure and the lack of a control group undermined the statistical power of the study. Pediatric population were excluded due to differences in the presentation of the disease and subsequent treatment regimens. The number of individuals employed in the study was decreased due to rare presentation of the disease. Regimens where chemotherapy was given as an adjuvant or a neoadjuvant to resection were only considered to maintain uniformity of the research analysis. This, however, could have resulted in loss of articles that describe for the use of a potential chemotherapeutic drug in colorectal MANEC management.

\section{Conclusions}

Any chemotherapeutic treatment regimen that is focused towards the adeno-carcinomatous or neuroendocrine component will be partially or completely effective against the tumor subtype. However, neuroendocrine tumors are highly aggressive and it is suggested that an adjuvant therapy focused on the neuroendocrine component of the tumor (for example: platinum-based combinations or streptozocin therapy) is administered as a first-line adjuvant therapy. In the current scenario, fluorouracil-based treatment regimens such as FLOT, FOLFOX, mFOLFOX are commonly employed in the neoadjuvant setting due to the antimetabolic activity of fluorouracil compounds and its high survival rate. FOLFOX is recommended due to decreased risk of adverse effects and similar effectiveness to FLOT. XELOX and platinum-based regimens have been initiated in the adjuvant setting with excellent responsiveness while streptozocin therapy shows great potential as an effective regimen in the management of colorectal MANEC.

\section{Additional Information \\ Disclosures}

Conflicts of interest: In compliance with the ICMJE uniform disclosure form, all authors declare the following: Payment/services info: All authors have declared that no financial support was received from any organization for the submitted work. Financial relationships: All authors have declared that they have no financial relationships at present or within the previous three years with any organizations that might have an interest in the submitted work. Other relationships: All authors have declared that there are no other relationships or activities that could appear to have influenced the submitted work.

\section{References}

1. Komminoth P, Arnold R, Capella C, et al.: Neuroendocrine neoplasms of the appendix. WHO Classification of Tumours of the Digestive System. Bosman FT, Carneiro F, Hruban RH, Theise ND (ed): International Agency for Research on Cancer, Lyon, France; 2010. 126-128.

2. La Rosa S, Marando A, Sessa F, Capella C: Mixed adenoneuroendocrine carcinomas (MANECs) of the gastrointestinal tract: an update. Cancers (Basel). 2012, 4:11-30. 10.3390/cancers4010011

3. Paspala A, Machairas N, Prodromidou A, et al.: Management of MANEC of the colon and rectum: a comprehensive review of the literature. Mol Clin Oncol. 2018, 9:219-222. 10.3892/mco.2018.1649

4. La Rosa S, Marando A, Furlan D, Sahnane N, Capella C: Colorectal poorly differentiated neuroendocrine carcinomas and mixed adenoneuroendocrine carcinomas: insights into the diagnostic immunophenotype, assessment of methylation profile, and search for prognostic markers. Am J Surg Pathol. 2012, 36:601-611. 10.1097/PAS.0b013e318242e21c

5. Tanaka T, Kaneko M, Nozawa H, et al.: Diagnosis, assessment, and therapeutic strategy for colorectal mixed adenoneuroendocrine carcinoma. Neuroendocrinology. 2017, 105:426-434. 10.1159/000478743 
6. Ma F, Wang B, Xue L, et al.: Neoadjuvant chemotherapy improves the survival of patients with neuroendocrine carcinoma and mixed adenoneuroendocrine carcinoma of the stomach. J Cancer Res Clin Oncol. 2020, 146:2135-2142. 10.1007/s00432-020-03214-W

7. Qiu S, Pellino G, Warren OJ, Mills S, Goldin R, Kontovounisios C, Tekkis PP: Mixed adenoneuroendocrine carcinoma of the colon and rectum. Acta Chir Belg. 2018, 118:273-277. 10.1080/00015458.2018.1482697

8. Li M, Zhang Y, Yang Q: Gastric mixed adenoneuroendocrine carcinoma with extensive liver metastases treated with neoadjuvant chemotherapy and surgery. J Cancer Sci Clin Ther. 2020, 4:195-199. 10.26502/jcsct.5079064

9. Oneda E, Liserre B, Bianchi D, Rota L, Savelli G, Zorzi F, Zaniboni A: Diagnosis of mixed adenoneuroendocrine carcinoma (MANEC) after neoadjuvant chemotherapy for pancreatic and gastric adenocarcinoma: two case reports and a review of the literature. Case Rep Oncol. 2019, 12:434-442. 10.1159/000501200

10. Lin Z, Chen J, Guo Y: Efficacy of XELOX adjuvant chemotherapy for gastric mixed adenoneuroendocrine carcinoma: a case report. Medicine (Baltimore). 2019, 98:e16000. 10.1097/MD.0000000000016000

11. Gül-Klein S, Sinn M, Jurmeister PS, et al.: Two patients with rare mixed adenoneuroendocrine carcinomas of the rectum. SAGE Open Med Case Rep. 2018, 6:2050313X18758816. 10.1177/2050313X18758816

12. Shin SH, Kim SH, Jung SH, et al.: High-grade mixed adenoneuroendocrine carcinoma in the cecum: a case report. Ann Coloproctol. 2017, 33:39-42. 10.3393/ac.2017.33.1.39

13. Tagai N, Goi T, Morikawa M, et al.: Favorable response of colonic mixed adenoneuroendocrine carcinoma to streptozocin monotherapy. Int Cancer Conf J. 2017, 6:175-179. 10.1007/s13691-017-0301-2

14. Silva JR, Pinho RT, Furtado A: A case of a mixed adenoneuroendocrine tumor of the colon . Rev Esp Enferm Dig. 2017, 109:673. 10.17235/reed.2017.5008/2017

15. Morais M, Pinho AC, Marques A, et al.: Mixed adenoneuroendocrine carcinoma causing colonic intussusception. Case Rep Surg. 2016, 2016:7684364. 10.1155/2016/7684364

16. Gurzu S, Kadar Z, Bara T, Bara T Jr, Tamasi A, Azamfirei L, Jung I: Mixed adenoneuroendocrine carcinoma of gastrointestinal tract: report of two cases. World J Gastroenterol. 2015, 21:1329-1333. 10.3748/wig.v21.i4.1329

17. Minaya-Bravo AM, Garcia Mahillo JC, Mendoza Moreno F, Noguelares Fraguas F, Granell J: Large cell neuroendocrine - Adenocarcinona mixed tumour of colon: Collision tumour with peculiar behaviour. What do we know about these tumours?. Ann Med Surg (Lond). 2015, 4:399-403. 10.1016/j.amsu.2015.10.004

18. Vanacker L, Smeets D, Hoorens A, et al.: Mixed adenoneuroendocrine carcinoma of the colon: Molecular pathogenesis and treatment. Anticancer Res. 2014, 34:5517-5521.

19. Yamauchi H, Sakurai S, Tsukagoshi R, et al.: A case of very well-differentiated adenocarcinoma with carcinoid tumor in the ascending colon. Int Surg. 2014, 99:132-136. 10.9738/INTSURG-D-13-00041.1

20. Ito H, Kudo A, Matsumura S, et al.: Mixed adenoneuroendocrine carcinoma of the colon progressed rapidly after hepatic rupture: report of a case. Int Surg. 2014, 99:40-44. 10.9738/INTSURG-D-13-00161.1

21. Koletsa T, Beretouli E, Tziola T, Mavropoulou S, Karayannopoulou G: Neuroendocrine carcinoma in adenoma of the sigmoid. Hippokratia. 2014, 18:362-363.

22. Jain A, Singla S, Jagdeesh KS, Vishnumurthy HY: Mixed adenoneuroendocrine carcinoma of cecum: a rare entity. J Clin Imaging Sci. 2013, 3:10. 10.4103/2156-7514.107995

23. Liu XJ, Feng JS, Xiang WY, Kong B, Wang LM, Zeng JC, Liang YF: Clinicopathological features of an ascending colon mixed adenoneuroendocrine carcinoma with clinical serosal invasion. Int J Clin Exp Pathol. 2014, 7:6395-6398.

24. Araki T, Takashima A, Hamaguchi T, et al.: Amrubicin in patients with platinum-refractory metastatic neuroendocrine carcinoma and mixed adenoneuroendocrine carcinoma of the gastrointestinal tract. Anticancer Drugs. 2016, 27:794-799. 10.1097/CAD.0000000000000393

25. Lin JP, Zhao YJ, He QL, et al.: Adjuvant chemotherapy for patients with gastric neuroendocrine carcinomas or mixed adenoneuroendocrine carcinomas. Br J Surg. 2020, 107:1163-1670. 10.1002/bjs.11608

26. Song LJ, Yuan L: Comparative analysis of colorectal mixed adenoneuroendocrine carcinoma and adenocarcinoma with neuroendocrine differentiation: a population-based study. Int J Clin Exp Pathol. 2019, 12:922-932.

27. van der Veen A, Seesing MF, Wijnhoven BP, et al.: Management of resectable esophageal and gastric (mixed adeno)neuroendocrine carcinoma: a nationwide cohort study. Eur J Surg Oncol. 2018, 44:1955-1962. 10.1016/j.ejso.2018.07.058

28. Komatsubara T, Koinuma K, Miyakura Y, et al.: Endocrine cell carcinomas of the colon and rectum: a clinicopathological evaluation. Clin J Gastroenterol. 2016, 9:1-6. 10.1007/s12328-015-0623-6

29. Watanabe J, Suwa Y, Ota M, et al.: Clinicopathological and prognostic evaluations of mixed adenoneuroendocrine carcinoma of the colon and rectum: a case-matched study. Dis Colon Rectum. 2016, 59:1160-1167. 10.1097/DCR.0000000000000702

30. Dulskas A, Pilvelis A: Oncologic outcome of mixed adenoneuroendocrine carcinoma (MANEC): a single center case series. Eur J Surg Oncol. 2020, 46:105-107. 10.1016/j.ejso.2019.08.002

31. Patta A, Fakih M: First-line cisplatin plus etoposide in high-grade metastatic neuroendocrine tumors of colon and rectum (MCRC NET): review of 8 cases. Anticancer Res. 2011, 31:975-978.

32. Maehara Y: S-1 in gastric cancer: a comprehensive review . Gastric Cancer. 2003, 6:2-8. 10.1007/s10120-0030232-9

33. Sah BK, Zhang B, Zhang $\mathrm{H}$, et al.: Neoadjuvant FLOT versus SOX phase II randomized clinical trial for patients with locally advanced gastric cancer. Nat Commun. 2020, 11:6093. 10.1038/s41467-020-19965-6

34. Yoshimura K, Uehara K, Tojima Y, et al.: Optimal schedule of adjuvant chemotherapy with S-1 for stage III colon cancer: study protocol for a randomized controlled trial. Trials. 2013, 14:17. 10.1186/1745-6215-14-17

35. Shitara K, Chin K, Yoshikawa T, et al.: Phase II study of adjuvant chemotherapy of S-1 plus oxaliplatin for patients with stage III gastric cancer after D2 gastrectomy. Gastric Cancer. 2017, 20:175-181. 10.1007/s10120-015-0581-1

36. National Comprehensive Cancer Network. NCCN Clinical Practice Guidelines in Oncology (NCCN Guidelines): neuroendocrine and adrenal tumors. (2021). Accessed: January 15, 2021: 


\section{Cureus}

http://www.nccn.org/professionals/physician_gls/pdf/neuroendocrine.pdf.

37. Cassidy J, Clarke S, Díaz-Rubio E, et al.: XELOX vs FOLFOX-4 as first-line therapy for metastatic colorectal cancer: NO16966 updated results. Br J Cancer. 2011, 105:58-64. 10.1038/bjc.2011.201

38. André T, Iveson T, Labianca R, et al.: The IDEA (International Duration Evaluation of Adjuvant Chemotherapy) collaboration: prospective combined analysis of phase III trials investigating duration of adjuvant therapy with the FOLFOX (FOLFOX4 or modified FOLFOX6) or XELOX (3 versus 6 months) regimen for patients with stage III colon cancer: trial design and current status. Curr Colorectal Cancer Rep. 2013, 9:261-269. 10.1007/s11888-013-0181-6

39. Ito M, Hirano Y, Isii T, et al.: Grade 3 well-differentiated neuroendocrine tumor of the rectum: a case report . Surg Case Rep. 2020, 6:130. 10.1186/s40792-020-00893-y

40. Plöckinger U, Rindi G, Arnold R, et al.: Guidelines for the diagnosis and treatment of neuroendocrine gastrointestinal tumours. A consensus statement on behalf of the European Neuroendocrine Tumour Society (ENETS). Neuroendocrinology. 2004, 80:394-424. 10.1159/000085237

41. Janson ET, Sorbye H, Welin S, et al.: Nordic guidelines 2014 for diagnosis and treatment of gastroenteropancreatic neuroendocrine neoplasms. Acta Oncol. 2014, 53:1284-1297. 10.3109/0284186X.2014.941999

42. Ito T, Masui T, Komoto I, et al.: The Clinical Guidelines for Pancreatic and Gastroenteric Neuroendocrine Tumors in Japan. Japan Neuroendocrine Tumor Society (ed): Kanehara Co Ltd, Tokyo; 2015.

43. Krug S, Boch M, Daniel H, et al.: Streptozocin-based chemotherapy in patients with advanced neuroendocrine neoplasms -- predictive and prognostic markers for treatment stratification. PLoS One. 2015, 10:e0143822. 10.1371/journal.pone.0143822

44. Aoki T, Kokudo N, Komoto I, et al.: Streptozocin chemotherapy for advanced/metastatic well-differentiated neuroendocrine tumors: an analysis of a multi-center survey in Japan. J Gastroenterol. 2015, 50:769-775. 10.1007/s00535-014-1006-3 